\title{
Clinical Profile of Respiratory Distress in Newborn in Chhattisgarh, India
}

\author{
Ashish Wanare ${ }^{1}$, Pradeep Agrawal ${ }^{1}$, Guruveerajeysingh Malini ${ }^{1}$, Mitesh Chawda ${ }^{1}$, Ganpat Jha ${ }^{1}$, \\ Ravi Pandey ${ }^{1}$, Bhushan Khadse ${ }^{2}$, Hitav Someshwar ${ }^{3}$, Akshay Patil ${ }^{4}$ \\ ${ }^{1}$ Department of Paediatrics, Jawaharlal Nehru Hospital \& Research Center, Bhilai, Chhattisgarh, India \\ ${ }^{2}$ Department of Radiology, Jawaharlal Nehru Hospital \& Research Center, Bhilai, Chhattisgarh, India \\ ${ }^{3}$ Department of Neurophysiotherapy, KJ Somaiya College of Physiotherapy, Mumbai, Maharashtra, India \\ ${ }^{4}$ Departmnet of Paediatrics, Dr. Vasantrao Pawar Medical College and Hospital, Nashik, Maharashtra, India
}

Email address:

chawdamitesh@gmail.com (M. Chawda)

\section{To cite this article:}

Ashish Wanare, Pradeep Agrawal, Guruveerajeysingh Malini, Mitesh Chawda, Ganpat Jha, Ravi Pandey, Bhushan Khadse, Hitav Someshwar, Akshay Patil. Clinical Profile of Respiratory Distress in Newborn in Chhattisgarh, India. European Journal of Clinical and Biomedical Sciences. Vol. 6, No. 3, 2020, pp. 35-42. doi: 10.11648/j.ejcbs.20200603.12

Received: May 13, 2020; Accepted: June 19, 2020; Published: August 10, 2020

\begin{abstract}
Present study was a Hospital based observational study of 100 newborns admitted in level II NICU. The study was done to assess the development of severe distress against onset, duration, oxygen requirement $\&$ outcome in terms of final diagnosis, mortality \& treatment intervention. Among the 100 cases, $90 \%$ cases were of respiratory in origin. Commonest cause of respiratory distress was transient tachypnoea of newborn followed by Meconium aspiration syndrome \& Respiratory distress syndrome of newborn. The onset of respiratory distress in newborn developing after 6 hours of birth \& respiratory distress $>24$ hours duration had severe respiratory distress. Newborn with risk factors like high maternal age, primigravida, $>4$ PV examinations, Meconium stained liquor and lower socioeconomic strata developed severe respiratory distress. Statistically significant correlation of severity of respiratory distress was not found with the mode of the delivery, Apgar score $<7$ at $1 \mathrm{~min}$, gestational age of the baby, birth weight and sex of the newborn. Only neonates with RDS \& MAS required ventilator care. Two of the patients of RDS required only surfactant therapy, while other two required surfactant with ventilator support. Amongst the 100 newborns with respiratory distress, mortality was in 5 newborn (5\%) which includes 2 of Respiratory Distress Syndrome \& 3 of Meconium Aspiration Syndrome.
\end{abstract}

Keywords: Respirtory Distress Syndrome, Neonates, Hylaline Membrane Disease, MAS

\section{Introduction}

The first breath and cry have always been mystical, signaling the beginning of new life. Variety of disorders of respiratory system and non respiratory disorders like intracranial injury, congenital malformations, metabolic disorders, septicemia and cardiac failure, can manifest clinically with respiratory distress. Improved early diagnosis and treatment due to technological advancements and increased neonatal specialization have led to a significant fall in neonatal mortality.

Ventilators have improvised the outcome of respiratory failure in neonates. Earlier, therapies for respiratory diseases were aimed at delivery of high concentration of $\mathrm{O}_{2}$. Now newer therapies are aimed at alleviating the physiological abnormalities of an immature or diseased lung while avoiding potentially harmful level of oxygen and positive pressure ventilatory support. Nonetheless, continued high incidence of premature birth and infants receiving poor prenatal care continues to test the abilities of the neonatologists. Early recognition and appropriate therapy of neonatal respiratory disease has got impressive results. Continued efforts in prevention of premature birth, early recognition of fetal distress, maternal risk factors for neonatal sepsis and diagnosis of diseases in utero will lead to further improvements in the neonatal outcome. Respiratory distress is diagnosed clinically by the presence of at least 2 of the following criteria namely, respiratory rate of $>60$ /minute, 
retractions (subcostal, xiphoid and suprasternal recession), flaring of alae nasi, expiratory grunt and cyanosis at room air on 2 consecutive examinations at least 1 hour apart. [1] The incidence may vary from $7-8 \%$ amongst live births. [2] Certain risk factors for developing respiratory distress includes prematurity, meconium-stained amniotic fluid (MSAF), caesarian section delivery, gestational diabetes, maternal chorioamnionitis, or prenatal ultrasonographic findings, such as oligohydramnios or structural lung abnormalities. [3-7]. The New born chest has limitation when compared to the adult chest. These limitations are explained else where. $[9,10]$ The cause of distress may be associated with the respiratory system or outside. Pulmonary causes account for $80-85 \%$ cases of respiratory distress in a newborn. [8]

Pulmonary causes include Transient tachypnea of newborn, Hyaline membrane disease, Meconium aspiration syndrome, Pneumonia, Pneumothorax, Congenital lung cysts, Diaphragmatic hernia, Choanal atresia \& Laryngeal web/ polyp. Extra-pulmonary cause include cardiac failure, anemia, polycythemia, septicemia, metabolic disorders, renal failure, renal tubular acidosis, meningitis/ intracranial bleed $\&$ ascites. We aimed to assess the various risk factors associated with development of severe respiratory distress in the new born and also to assess the immediate clinical outcome of respiratory distress in newborn.

\section{Methodology}

We conducted a cross sectional, prospective observational study to assess clinical profile of respiratory distress in newborn at a LEVEL-II Neonatal Intensive Care Unit in a territory care hospital for a duration of 1 year with a sample size calculated at 100 subjects. We used a pre designed, pretested proforma for assessing our subjects. We had taken ethical clearance from the Ethical committee of our institution. We had taken informed consent from parents of neonate which passed our criteria for inclusion. We included those neonates with respiratory distress within 72 hours of life irrespective of weight, gestation age, mode of delivery \& Sex. The written informed consent was taken by all the study participant's parents. Data was collected for all newborns included in the study with respiratory distress such as general information, socioeconomic status, history and clinical examination findings of mother and newborn. Newborn with respiratory distress were shifted to NICU for further management. Time of onset of distress was documented and the severity of the distress was documented and the severity was assessed by using Silverman \& Anderson in preterms and Downs clinical scoring in term infants [9]. Serial x-rays were done in all newborns and were reported by the radiologist for abnormal findings. Sp02 monitoring was done and depending on the clinical diagnosis of respiratory distress, relevant investigations were sent such as Complete Blood Count, CRP, RBS, Sr. Calcium, Blood
$\mathrm{C} / \mathrm{S}$ and Chest X-ray and newborns were managed as per protocol. Duration and quantification of $\mathrm{O}_{2}$ therapy, intervention done in the form of surgical / ventilator / surfactant therapy/ treatment and mortality was documented to assess the clinical outcome against the final diagnosis.

Statistical analysis: Data was compiled in Ms-excel and was checked for its completeness and correctness. Then it was analyzed by using suitable statistical software (SPSS17.0). All means are expressed as mean \pm standard deviation. Data was presented in form of number and percentage. Qualitative and quantitative data was analyzed using Chi-square test and T test respectively. $\mathrm{P}$ value $<0.05$ is considered as a statistical significance.

\section{Results}

(I) Clinical Assesment of Outcome of Respiratory Distress In Newborn

Table 1. System wise distribution of etiology of respiratory distress.

\begin{tabular}{lll}
\hline Etiology & Frequency & Percentage \\
\hline RS & 90 & $90 \%$ \\
CVS & 5 & $5 \%$ \\
SEPSIS & 5 & $5 \%$ \\
TOTAL & 100 & $100 \%$ \\
\hline
\end{tabular}

It was seen that in $90 \%$ of the cases of newborn respiratory distress the cause was respiratory in origin. There were $5 \%$ cases of sepsis \& 5\% cases of Congenital heart disease (Acyanotic CHD, VSD, ASD, PDA, moderate PAH, heart failure).

Table 2. Etiology of respiratory distress.

\begin{tabular}{ll}
\hline Etiology & $\mathbf{N}(\%)$ \\
\hline TTNB & 58 \\
MAS & 20 \\
RDS & 12 \\
Sepsis & 5 \\
CHD & 5 \\
Total & 100 \\
\hline
\end{tabular}

Among the causes of respiratory distress, $58 \%$ is due to transient tachyponea of newborn (TTNB), $20 \%$ due to meconium aspiration syndrome (MAS), 12\% because of respiratory distress syndrome (RDS), 5\% belongs to sepsis and rest $5 \%$ due to congenital heart disease (CHD).

Table 3. Severity of respiratory distress.

\begin{tabular}{llll}
\hline $\begin{array}{l}\text { Grading of Respiratory } \\
\text { Distress }\end{array}$ & $\begin{array}{l}\text { S-A } \\
\text { Score }\end{array}$ & Frequency & Percentage \\
\hline Mild & $<3$ & $10(10 \%)$ & 10 \\
Moderate & $3-7$ & $46(46 \%)$ & 46 \\
Severe & $>7$ & $44(44 \%)$ & 44 \\
Total & & 100 & 100 \\
\hline
\end{tabular}

Majority of the newborns had moderate respiratory distress (46\%) \& severe respiratory distress (44\%) compared to mild distress (10\%). 
Table 4. Final diagnosis vs severity of respiratory distress.

\begin{tabular}{llll}
\hline \multirow{2}{*}{ Final Diagnosis } & \multicolumn{2}{l}{ SEVERITY OF RESP DISTRESS } & \multirow{2}{*}{ Total } \\
\cline { 2 - 3 } & Mild \& Moderate & Severe & $58(100 \%)$ \\
\hline Transient Tachypnea of newborn (TTNB) & $39(67.24 \%)$ & $19(32.75 \%)$ & $20(100 \%)$ \\
Meconium Aspiration Syndrome (MAS) & $7(35 \%)$ & $13(65 \%)$ & $12(100 \%)$ \\
Respiratory Distress Syndrome (RDS) & $4(33.33 \%)$ & $8(66.66 \%)$ & $5(100 \%)$ \\
Sepsis & $2(40 \%)$ & $3(60 \%)$ & $5(100 \%)$ \\
CHD & $4(80 \%)$ & $1(20 \%)$ & 100 \\
Total & $56(56 \%)$ & $44(44 \%)$ & 100 \\
\hline
\end{tabular}

\begin{tabular}{lll}
\hline Chi square & P value & Significance \\
\hline 10.75 & 0.029 & SIGNIFICANT \\
\hline
\end{tabular}

$66.66 \%$ of newborns with respiratory distress diagnosed with RDS ( 8 out of 12 ), $65 \%$ diagnosed with MAS (13 out of 20 ) and $60 \%$ diagnosed with sepsis (3 out of 5) had developed severe respiratory distress as compared to $32.75 \%$ of the neonates with respiratory distress with diagnosis of TTNB (19 out of 58) and 20\% neonates diagnosed with CHD (1 OUT OF 5).

Above Distribution of final diagnosis between mild and moderate combined and severe respiratory distress is found to be significant. It means TTNB, CHD has more incidence of mild and moderate respiratory distress than severe. Similarly in MAS, RDS and SEPSIS has more incidence of severe respiratory distress than mild and moderate combined.

Table 5. Onset vs severity of respiratory distress.

\begin{tabular}{llll}
\hline \multirow{2}{*}{ Onset } & \multicolumn{2}{l}{ SEVERITY OF RESPIRATORY DISTRESS } & \multirow{2}{*}{ Total } \\
\cline { 2 - 3 } & Mild \& Moderate & Severe & \\
\hline At Birth & $35(56.4 \%)$ & $27(43.6 \%)$ & 62 \\
$0-6$ hours & $15(68.2 \%)$ & $7(31.8 \%)$ & 22 \\
$>6$ hours & $6(37.5 \%)$ & $10(62.5 \%)$ & 16 \\
Total & $56(56 \%)$ & $44(44 \%)$ & 100 \\
\hline
\end{tabular}

\begin{tabular}{lll}
\hline Chi square & p-value & Significance \\
\hline 3.25 & 0.17 & Not significant \\
\hline
\end{tabular}

$62.5 \%$ of the newborns (10 out of 16 ) with the onset of respiratory distress after 6 hours of birth developed severe distress compared to $43.6 \%$ (27 out of 62 ) \& $31.8 \%$ (7 out of 22) newborn with onset of respiratory distress at birth and between 0-6 hoursof birth respectively.

Above distribution of onset of respiratory distress at birth, 0-6 hours and $>6$ hours between mild and moderate combined and severe respiratory distress is found not significant. It means even though it seems onset of distress has more number of severe cases it has no statistical significance.

Table 6. Duration of respiratory distress vs severity of respiratory distress.

\begin{tabular}{llll}
\hline Duration of & \multicolumn{2}{l}{ Severity Respiratory Distress } & \multirow{2}{*}{ Total } \\
\cline { 2 - 3 } Respiratory Distress & Mild \& Moderate & Severe & \\
\hline$<24$ & $46(80.7 \%)$ & $11(19.3 \%)$ & 57 \\
$>24$ & $10(23.25 \%)$ & $33(76.75 \%)$ & 43 \\
Total & $56(56 \%)$ & $44(44 \%)$ & 100 \\
\hline & & & \\
\hline Chi square test & p-value & Significance \\
\hline 32.82 & $<0.0001$ & Highly significant \\
\hline
\end{tabular}

$76.75 \%$ of the newborns (33 out of 43 ) with duration of respiratory distress more than 24 hours developed severe respiratory distress compared to $19.3 \%$ of newborns (11 out of 57) with duration of less than 24 hours.

Above Distribution of duration of respiratory distress $<24$ hours and $>24$ hours with severity of respiratory distress is highly significant suggesting severity increases with duration of respiratory distress.

Table 7. Duration of oxygen therapy vs final diagnosis of respiratory distress.

\begin{tabular}{|c|c|c|c|c|}
\hline \multirow{2}{*}{ Final Diagnosis } & \multirow{2}{*}{$\mathbf{N}$} & \multirow{2}{*}{ Severe Distress } & \multicolumn{2}{|c|}{ Oxygen therapy } \\
\hline & & & $\leq 24$ hours & $>24$ hours \\
\hline TTNB & 58 & $19(32.76 \%)$ & $33(56.9 \%)$ & $25(43.1 \%)$ \\
\hline MAS & 20 & $13(65 \%)$ & $6(30 \%)$ & $14(70 \%)$ \\
\hline RDS & 12 & $8(66.67 \%)$ & $3(25 \%)$ & $9(75 \%)$ \\
\hline Sepsis & 5 & $3(60 \%)$ & $1(20 \%)$ & $4(80 \%)$ \\
\hline CHD & 5 & $1(20 \%)$ & $4(80 \%)$ & $1(20 \%)$ \\
\hline Total & 100 & $44(44 \%)$ & $47(47 \%)$ & $53(53 \%)$ \\
\hline Chi square & & P-value & \multicolumn{2}{|c|}{ Significance } \\
\hline 10.58 & & 0.03 & \multicolumn{2}{|c|}{ Significant } \\
\hline
\end{tabular}

Majority (53\%) of newborn with respiratory distress required oxygen treatment for more than 24 hours as compared to $47 \%$ of newborn with respiratory distress for less than 24 hours duration. Out of 58 newborns with TTNB, only 19 $(32.7 \%)$ developed severe respiratory distress and only $43.1 \%$ required oxygen for $>24$ hours. 13 out of 20 newborns of MAS $(65 \%) \& 8$ out of 12 newborn with RDS (66.67\%) developed severe distress and required oxygen more than 24 hours in all of them. Whereas in all $70 \%$ and $75 \%$ of newborns with MAS and RDS respectively required oxygen support for $>24$ hours. 
Above distribution of duration of respiratory distress and final diagnosis is found to be significant. $>24$ hours oxygen

requirement was more in MAS, RDS and sepsis whereas it was less in TTNB and CHD.

Table 8. Intervention.

\begin{tabular}{|c|c|c|c|c|c|}
\hline \multirow{2}{*}{ Final Diagnosis } & \multicolumn{4}{|c|}{ INTERVENTION } & \multirow{2}{*}{ TOTAL } \\
\hline & O2 SUPPORT & VENTILATION & SURFACTANT & VENTILATION \& SURFACTANT & \\
\hline TTNB & 58 & 0 & 0 & 0 & 58 \\
\hline MAS & 14 & 6 & 0 & 0 & 20 \\
\hline RDS & 8 & 0 & 2 & 2 & 12 \\
\hline Sepsis & 5 & 0 & 0 & 0 & 5 \\
\hline CHD & 5 & 0 & 0 & 0 & 5 \\
\hline Total & $90(90 \%)$ & $6(6 \%)$ & $2(2 \%)$ & $2(2 \%)$ & 100 \\
\hline
\end{tabular}

\begin{tabular}{lll}
\hline Chi-square test & p-value & Significance \\
\hline 55.70 & $<0.001$ & Significant \\
\hline
\end{tabular}

Among the different causes of respiratory distress $90 \%$ cases require only oxygen support, $6 \%$ cases require ventilator support, $2 \%$ required surfactant and $2 \%$ cases require ventilation and surfactant both.

Distribution of final diagnosis and intervention done in the cases is found to be significant. Suggestive of that all TTNB were managed on $\mathrm{O} 2$ support and did not require further intervention.

Table 9. Final diagnosis vs outcome.

\begin{tabular}{llll}
\hline \multirow{2}{*}{ Final Diagnosis } & Outcome & Discharge & TOTAL \\
\cline { 2 - 4 } & Death & 58 & 58 \\
TTNB & 0 & $17(85 \%)$ & 20 \\
MAS & $3(15 \%)$ & $10(83.33 \%)$ & 12 \\
RDS & $2(16.67 \%)$ & 5 & 5 \\
Sepsis & 0 & 5 & 5 \\
CHD & 0 & $95(95 \%)$ & 100 \\
Total & $5(5 \%)$ & & \\
\hline
\end{tabular}

\begin{tabular}{lll}
\hline Chi square & P value & Significance \\
\hline 11.23 & 0.024 & Significant \\
\hline
\end{tabular}

Distribution of final diagnosis with outcome that is death or discharge is significant. All patients having TTNB, CHD and sepsis were discharged with no mortality where as MAS and RDS had $15 \%$ (3 out of 20 ) and $16.67 \%$ ( 2 out of 12 ) deaths respectively.

(Ii) Risk Factor for Development of Severe Respiratory Distress

$63 \%$ of newborns (17 out of 27 ) from upper lower class of kuppuswami scale developed severe respiratory distress compared to $45.5 \%$ (15 out of 33 ) \& $30 \%$ (12 out of 40 ) newborn from lower middle \& upper middle class respectively. Above distribution of socioeconomic status with severity of respiratory distress is significant \& suggesting that Socioeconomic status had impact on severity of respiratory distress according to our study. Lower the socioeconomic strata, higher was the severity. Thus the distribution is significant. $80 \%$ of the newborns ( 8 out of 10 ) born to mothers with maternal age $>30$ developed severe respiratory distress compared to $55.5 \%$ (5 out of 9 ) and $38 \%$ (31 out of 81 ) newborns born to mothers below 21 years \& between 22-30 years respectively. Maternal age after being divided in $<21$ years, $22-30$ and $>30$ years $\&$ p-value being significant suggests that 22-30 year age group had more incidence of mild and moderate respiratory distress than severe one. Whereas severity increases on both extremes. $59.2 \%$ of the newborns ( 29 out of 49 ) born to Primigravida mothers developed severe respiratory distress compared to $29.4 \%$ (15 out of 51 ) newborns born to multi Gravida mother. p-value being significant in study clearly suggests more incidence of severe respiratory distress in primi than multigravida where mild and moderate distress was more common. $68.75 \%$ of the newborns (22 out of 32) born to mothers who had more than 4 Per vaginal examinations had severe respiratory distress compared to $32.35 \%$ (22 out of 68 ) born to mothers who had less than 4 per vaginal examinations. Distribution of PV examination with severity of distress is highly significant suggesting more number of PV examinations increases the risk of respiratory distress and its severity. $65 \%$ of newborns (13 out of 20 ) born to mothers with meconium stained liquor developed severe respiratory distress compared to $38.75 \%$ of the newborns ( 31 out of 80 ) born to mother with clear liquor. p-value is significant suggestive of Meconium stained liquor increases the risk of severity of respiratory. $39.29 \%$ of newborns (11 out of 28) born by cesarean section developed severe respiratory distress compared to $47.36 \%$ of the newborns ( 9 out of 19) born by normal vaginal route. Distribution between mode of delivery and the severity was not significant suggesting there was no correlation between mode of delivery whether NVD, LSCS or forceps with the severity of distress. $58.82 \%$ of preterm newborns (10 out of 17) developed severe respiratory distress compared to $40.96 \%$ (34 out of 83 ) 
among fullterm newborns. Though preterms had more incidence of severe respiratory distress in our study than in terms, the distribution is insignificant as our study had more number of term neonates with TTNB. $47.76 \%$ of newborns (32 out of 67) with1 min apgar of less than 7 developed severe respiratory distress compared to $36.36 \%$ (12 out of 33 ) with 1 min apgar more than 7 . Distribution between $1 \mathrm{~min}$ apgar score and the severity was not significant suggesting there was no correlation between $1 \mathrm{~min}$ apgar score with the severity of distress. $48.15 \%$ of the newborns (13 out of 27 ) with birth weight of $<2.5 \mathrm{Kgs}$ had developed severe respiratory distress compared to $40 \%$ (26 out of 65 ) \& $62.5 \%$ ( 5 out of 8 ) with birth weight of $2.5-3.5 \mathrm{Kgs} \&>3.5 \mathrm{kgs}$ respectively. Distribution between birth weight and the severity was not significant suggesting there was no correlation between birth weight with the severity of distress. $46.77 \%$ of the newborns (29 out of 62) male babies developed severe respiratory distress compared to $39.47 \%$ (15 out of 38) female babies. Distribution between newborn sex and the severity was not significant suggesting there was no correlation between newborn sex with the severity of distress.

\section{Discussion}

Early diagnosis of newborn distress is very important for its management and good clinical outcome. This study has made an attempt in early identification of the cause of newborn distress by clinical assessment of its severity, risk factor association \& abnormal radiological findings.

In the present study out of 100 cases identified with respiratory distress, $90 \%$ were respiratory in origin, $5 \%$ had congenital heart disease and 5\% had sepsis. Out of $90 \%$ of respiratory cases, 58\% had TTNB, 20\% had MAS and $12 \%$ had RDS. $44 \%$ of the newborn had severe respiratory distress while $46 \%$ had moderate respiratory distress \& $10 \%$ had mild respiratory distress. The commonest cause for respiratory distress was Transient Tachypnoea of Newborn (58\%) followed by Meconium Aspiration Syndrome (20\%) \& RDS (12\%). No surgical cause like $\mathrm{CDH}$ was found during study. Similar result was seen in the study done by Guyon G [4] where the commonest cause for respiratory distress in newborns was TTNB (72\%) followed by MAS (61\%) \& RDS (38\%). However in a study done by Alok Kumar [8] it was seen that the RDS was found to be the commonest $(42.7 \%)$ cause of respiratory distress followed by TTNB $(17.0 \%)$, MAS (10.7\%), Sepsis (9.3\%) and birth asphyxia $(3.3 \%)$. Similarly Nagendra K [10] also shows that the commonest cause for respiratory distress in neonates was RDS (18.8\%) followed by TTNB (14\%) and MAS (12.5\%). This variability in the present study was due to increased no of cesarean deliveries during the study period giving rise to more no of TTNB cases.

$62.5 \%$ of the newborns ( 10 out of 16 ) with the onset of respiratory distress after 6 hours of birth developed severe distress compared to $43.6 \%$ (27 out of 62 ) \& $31.8 \%$ (7 out of 22) newborn with onset of respiratory distress at birth and between 0-6 hours of birth respectively. Similar results were seen in a study done by Michal Rygl [11], neonates with onset of respiratory distress after 6 hours, the survival rate was found to be $45.3 \%$.

In the present study it was seen that $76.75 \%$ of the newborns (33 out of 43) with duration of respiratory distress more than 24 hours developed severe respiratory distress compared to neonates which were diagnosed with the onset of respiratory distress at birth. Similar results were observed in the study done by Paediatrician in America [12] where neonates (34 out of 71) with the duration of respiratory distress of more than 24 hours developed severe respiratory distress. In our study, majority (53\%) required $\mathrm{O}_{2}$ more than 24 hours and 43 out of 44 severe distress cases required $\mathrm{O}_{2}$ support for $>24$ hours which implied $\mathrm{O}_{2}$ requirement depends on the severity of respiratory distress. A research team [13] studied the neonates born with respiratory distress requiring supplemental oxygen and it was seen that $8 \%$ required supplemental oxygen for at least an hour. The discrepancy in the present study may be due to more number of TTNB $(58 \%)$ which do not require more $\mathrm{O}_{2}$ due to less severity of distress. On the contrary newborns with MAS (70\%) \& RDS (75\%) required more $\mathrm{O}_{2}$ as they tend to develop more severe distress. Bhutta ZA [14] studied 200 babies born out of which 81 were diagnosed with respiratory distress. It was seen that these babies required supplemental oxygen while in NICU. But unlike our study the above study has not analyzed $\mathrm{O}_{2}$ requirement depending on the cause of respiratory distress which would help to determine clinical outcome.

$90 \%$ of the patients were managed exclusively with oxygen support. Surgical intervention was not required in any case. 6 out of 20 cases of MAS required ventilation support, 2 out of 12 patients of RDS required surfactant therapy and 2 required ventilation and surfactant. Rest of the patients were recovered with exclusive oxygen support. This suggests that oxygen support was all that was needed in TTNB Patil A [15] has done similar study in 78 newborns with respiratory distress where he got similar findings. One newborn had $\mathrm{CDH}$ and required surgical intervention and ventilator support where as four cases of MAS and 2 of RDS needed ventilator support. One case with RDS required surfactant therapy.

There were five mortalities seen in our 100 newborn cases with respiratory distress. We had no mortality in TTNB, $\mathrm{CHD}$ and sepsis affected newborns. Most of the cases of TTNB recovered within 48-72 hours and all were discharged. 3 out of $20(15 \%)$ cases of MAS succumbed to illness and 2 out of $12(16.67 \%)$ cases died due to RDS. Mortality in our study is $5 \%$ which is much low in other studies done elsewhere. Shrivastava S. P.[16] studied 1000 babies and found the most common respiratory cause of neonatal death was due to birth Asphyxia (44\%), followed by RDS (4.4\%), $\operatorname{MAS}(1.9 \%)$.

\subsection{Maternal Risk Factors}

\subsubsection{Socioeconomic Status}

In the present study, socioeconomic status was graded by 
modified kuppuswami scale. 63\% (17 out of 27) cases in upper lower class developed severe respiratory distress compared to $30 \%(12$ out of 40$)$ in upper middle and $45.5 \%$ (15 out of 33 ) in lower middle class developed severe respiratory distress. Kommawar A [17]. has similar observation like our study suggesting highly significant association between lower socioeconomic status and severity of respiratory distress.

\subsubsection{Maternal Age}

In the present study it was seen that $80 \%$ of the newborns ( 8 out of 10) born to mothers with maternal age $>30$ developed severe respiratory distress compared to $55.5 \%(5$ out of 9 ) and $38 \%$ (31 out of 81 ) newborns born to mothers below 21 years $\&$ between 22-30 years respectively. Similar results were observed in the study done by C. Dani [18] where it was observed that mothers $>32$ years of age were at a higher risk of delivering babies with respiratory distress.

Similar comparison was seen in the study done by Alwyn Smith [19] were higher maternal age was identified as a risk factor for neonatal respiratory distress. In the present study it was observed higher maternal age to be a risk factor for developing respiratory distress in newborns as the correlation is statistically significant.

\subsubsection{Parity}

In the present study $59.2 \%$ of the newborns (29 out of 49 ) born to Primigravida mothers developed severe respiratory distress when compared to $29.4 \%$ (15 out of 51 ) newborns born to multigravida. Similar results were observed in the study done by C. Dani [18] where it was seen that the incidence of respiratory distress was more in the first pregnancy and after the fourth pregnancy. However N. B. Mathur [20] has shown that multigravida was a risk factors for developing respiratory distress in newborns. M. Lureti [21] however shows that there is no significant association between multiparity and the attainment of respiratory distress. Prolonged labour in the primigravida may be the reason for development of respiratory distress in neonates in the present study.

\subsubsection{PV Examination}

Bertini [22] has shown that the number of PV examination above 4 was significantly associated with respiratory distress. Similar results were observed in the study done by Kwang sun Lee et al [23] where per vaginal examination done more than 5 before the delivery time was more associated with respiratory distress. $68.75 \%$ of the newborns (22 out of 32) born to mothers who had more than 4 Per vaginal examinations had severe respiratory distress compared to $32.35 \%$ (22 out of 68 ) born to mothers who had less than 4 per vaginal examinations M. F. Reali [24]. This suggests that higher the number of per vaginal examinations more is the newborn prone to infection and in turn to severe respiratory distress.

\subsubsection{Liquor}

$65 \%$ of newborns (13 out of 20 ) born to mothers with meconium stained liquor developed severe respiratory distress compared to $38.75 \%$ of the newborns ( 31 out of 80 ) born to mother with clear liquor. Similar observations were observed in the study done by Michal Rygal [5] where meconium stained liquor had more chances of developing respiratory distress $58.8 \%$ when compared to $21.14 \%$.

\subsubsection{Mode of Delivery}

$39.29 \%$ of newborns (11 out of 28) born by cesarean section developed severe respiratory distress compared to $47.36 \%$ of the newborns (33 out of 69 ) born by normal vaginal route. In our study, mode of delivery and severity of respiratory distress was not statistically significant. Same parameter was observed in studies done by C. Dani [18], EJ Gellee [25] \& Jean-Bernard Gouyon [4] where it was noticed that caesarian delivered babies have more chances of neonatal respiratory distress when compared to normal vaginal delivery.

\subsection{Fetal Risk Factors}

\subsubsection{Gestational Age}

In the present study it was seen that $58.82 \%$ of newborns (10 out of 17) with SGA developed severe respiratory distress compared to $40.96 \%$ (34 out of 83) with term gestational age. Most of the studies like as done by Kwang sun Lee et al [23] where it was seen that SGA babies had a significantly higher association with the incidence of respiratory distress when compared to term babies. C. Dani [18] \& M. Lureti [21] has also observed in their studies that SGA babies were more associated with respiratory distress when compared to term babies. In our study, though preterm babies have higher percentage of severe respiratory distress, it is not statistically significant. It is probably because our study has more number of term babies with maximum number being TTNB.

\subsubsection{One Min Apgar Score}

In the present study it was seen that $47.76 \%$ of newborns (32 out of 67) with1 min apgar of less than 7 developed severe respiratory distress compared to $36.36 \%$ (12 out of 33) with 1 min apgar more than 7 . Study done by M. Lureti [21] has shown that a Low Apgar score at 1 st and 5 th $\min <7$ was associated with increased risk of respiratory distress when compared to babies with Apgar score more than 7.9 at 1 minute of age and 8.4 for 5 minutes of age.

Similarly Fidanovski. D [26] observed that low $1 \mathrm{~min}$ apgar score was more associated with respiratory distress and Prolonged NICU stay. However Jean-Bernard Gouyon [4] showed that Apgar score $\leq 3$ at 1 min was more associated with increased respiratory distress. In our study $1 \mathrm{~min}$ APGAR score of $<7$ has higher percentage of severe respiratory distress but the statistical significance is lacking.

\subsubsection{Birth Weight}

In the present study it was seen that $48.15 \%$ of the newborns (13 out of 27) with birthweight of $<2.5 \mathrm{Kg}$ had developed severe respiratory distress compared to $40 \%$ (26 out of 65 ) \& $62.5 \%$ (5 out of 8) with birth weight of $2.5-3.5$ $\mathrm{Kg} \&>3.5 \mathrm{~kg}$ respectively. In the study done by M. Lureti [21] 
it was seen that the risk of neonatal respiratory distress markedly increased with decreasing birth weight compared to babies weighing more than $2500 \mathrm{~g}$ at birth. However in the study done by Herbert C Miller [27] it was seen that respiratory distress was more common in babies weighing between $1000 \mathrm{gms}-1500 \mathrm{gms}$ and the risk of respiratory distress reduced with increase in birth weight. In our study, birth weight is not statistically correlated with severity of respiratory distress probably because of higher number of term babies with good birth weight and most of them having TTNB.

\subsubsection{Baby Sex}

In the present study it was seen that $46.77 \%$ of the newborns (29 out of 62) male babies developed severe respiratory distress compared to $39.47 \%$ (15 out of 38 ) female babies. M. Lureti [21] shows the frequency of neonatal respiratory distress was higher in males than compared with females. Similarly Herbert C Miller [27] \& Sengupta [28] shows that the incidence of severe respiratory distress was almost three times higher among males than females However in the study done by C. Dani [18] it was observed that that there is no significant association with respiratory distress and the sex of the baby. Similar observations were observed by Negendra K [10] who also concluded that there is no significant difference in neonatal respiratory distress in male and female neonates.

\section{Conclusions}

There are very few studies on comparison of etiology with the severity of the respiratory distress. We found an overall survival rate was $95 \%$. Transient tachypnea of the newborn was the most common cause of respiratory distress in newborn followed by MAS followed by RDS. 44\% of newborn with respiratory distress developed severe respiratory distress which required intensive monitoring. Risk factors like high maternal age, primigravida mothers, more than 4 per vaginal examinations, meconium stained liquor, low socioeconomic status were associated with severe respiratory distress in newborns. In our study, mode of delivery, gestational age, 1 min APGAR Score $<7$, birth weight and newborn sex had no correlation with severity of respiratory distress. Clinical assessment of severe respiratory distress against its onset and duration will help in early diagnosis. Immediate clinical outcome of newborn respiratory distress in term of mortality rate is variable and depends on the cause of newborn distress.

\section{Recommendation}

1) Appropriate ANC care with early recognition and timely follow up of high risk cases and prompt management is required to reduce morbidity.

2) Aseptic handling with minimal PV examinations wherever possible is recommended.

3) Modifiable risk factors associated with low socioeconomic status like anemia, undernutrition and hygiene must be dealt with to reduce adverse impact on newborn.

4) Timely recognition and intervention of respiratory distress is recommended to reduce morbidity and mortality.

5) Silverman Anderson and Downe scoring system being manual methods and do not require any instruments, assessment of the severity of respiratory distress can be done even in resource poor places.

\section{References}

[1] Meharban Singh, Care of the newborn $7^{\text {th }}$ edition: 273-83.

[2] Edwards MO, Kotecha SJ, Kotecha S. Respiratory distress of the term newborn infant. Paediatric respiratory reviews. 2013 Mar 1; 14 (1): 29-37.

[3] Hibbard JU, Wilkins I, Sun L, Gregory K, Haberman S, Hoffman M, Kominiarek MA, Reddy U, Bailit J, Branch DW, Burkman R. Respiratory morbidity in late preterm births. JAMA: the journal of the American Medical Association. 2010 Jul 28; 304 (4): 419.

[4] Gouyon JB, Ribakovsky C, Ferdynus C, Quantin C, Sagot P, Gouyon B, Burgundy Perinatal Network. Severe respiratory disorders in term neonates. Paediatric and perinatal epidemiology. 2008 Jan; 22 (1): 22-30.

[5] Williams O, Hutchings $G$, Hubinont $C$, Debauche $C$, Greenough A. Pulmonary effects of prolonged oligohydramnios following mid-trimester rupture of the membranes-antenatal and postnatal management. Neonatology. 2012; 101 (2): 83-90.

[6] Jobe AH. Effects of chorioamnionitis on the fetal lung. Clinics in perinatology. 2012 Sep 1; 39 (3): 441-57.

[7] Bak SY, Shin YH, Jeon JH, Park KH, Kang JH, Cha DH, Han MY, Jo HS, Lee KH, Lee CA. Prognostic factors for treatment outcomes in transient tachypnea of the newborn. Pediatrics International. 2012 Dec; 54 (6): 875-80.

[8] Kumar A, Bhat BV. Respiratory distress in newborn. Indian Journal of Maternal and Child Health. 1996; 7 (1): 8-10.

[9] Mathai SS, Raju U, Kanitkar M. Management of respiratory distress in the newborn. Medical journal, Armed Forces India. 2007 Jul; 63 (3): 269.

[10] Nagendra K, Wilson CG, Ravichander B, Sood S, Singh SP: incidence and etiology of respiratory distress in newborn Medical Journal Armed Forces India. 1999 Oct; 55 (4): 3313.

[11] Sane SY, Patel BM. Neonatal respiratory distress syndrome: an autopsy study of 190 cases. The Indian Journal of Pediatrics. 1985 Jan 1; 52 (1): 43-6.

[12] Angus DC, Linde-Zwirble WT, Clermont G, Griffin MF, Clark RH. Epidemiology of neonatal respiratory failure in the United States: projections from California and New York. American journal of respiratory and critical care medicine. 2001 Oct 1; 164 (7): 1154-60.

[13] Cloherty JP, Eichenwald EC, Stark AR: Manual of neonatal care: $7^{\text {th }}$ Edition: Lippincott Williams \& Wilkins; 2012. 
[14] Bhutta ZA, Yusuf K. Neonatal respiratory distress syndrome in Karachi: some epidemiological considerations. Paediatric and perinatal epidemiology. 1997 Jan; 11 (1): 37-43.

[15] Patil AN., Sonawane S., Ahire N., Clinical Profile Of Respiratory Distress In Newborn. MVPJS. 2018 June (5) 19735.

[16] Shrivastava SP, Kumar A, Ojha AK. Verbal autopsy determined causes of neonatal deaths. Indian pediatrics. 2001 Sep 1; 38 (9): 1022-4.

[17] Kommawar A, Borkar R, Vagha J, Lakhkar B, Meshram R, Taksandae A. Study of respiratory distress in newborn. International Journal of Contemporary Pediatrics. 2017 Feb 22; 4 (2): 490-4.

[18] Dani C, Reali MF, Bertini G, Wiechmann L, Spagnolo A, Tangucci M, Rubaltelli FF. Risk factors for the development of respiratory distress syndrome and transient tachypnoea in newborn infants. Italian Group of Neonatal Pneumology. European Respiratory Journal. 1999 Jul 1; 14 (1): 155-9.

[19] Greer JJ. Current concepts on the pathogenesis and etiology of congenital diaphragmatic hernia. Respir Physiol Neurobiol. 2013; 189 (2): 232-240. Doi: 10.1016/j.resp.2013.04.015.

[20] Mathur NB, Garg K, Kumar S. Respiratory distress in neonates with special reference to pneumonia. Indian pediatrics. 2002 Jun 27; 39 (6): 529-38.

[21] Luerti M, Parazzini F, Agarossi A, Bianchi C, Rocchetti M, Bevilacqua G. Risk factors for respiratory distress syndrome in the newborn: a multicenter Italian survey. Acta obstetricia et gynecologica Scandinavica. 1993 Jul; 72 (5): 359-64.

[22] Rubaltelli FF, Dani C, Reali MF, Bertini G, Wiechmann L,
Tangucci M, Spagnolo A, Italian Group of Neonatal Pneumology. Acute neonatal respiratory distress in Italy: a one-year prospective study. Acta paediatrica. 1998 Dec; 87 (12): $1261-8$.

[23] Lee KS, Eidelman AI, Tseng PI, Kandail SR, Gartner LM. Respiratory distress syndrome of the newborn and complications of pregnancy. Pediatrics. 1976 Nov 1; 58 (5): 675-80.

[24] McElhatton PR, Garbis HM, Eléfant E, Vial T, Bellemin B, Mastroiacovo P, Arnon J, Rodríguez-Pinilla E, Schaefer C, Pexieder T, Merlob P. The outcome of pregnancy in 689 women exposed to therapeutic doses of antidepressants. A collaborative study of the European Network of Teratology Information Services (ENTIS). Reproductive toxicology. 1996 Jul 1; 10 (4): 285-94.

[25] Geller EJ, Wu JM, Jannelli ML, Nguyen TV, Visco AG. Neonatal outcomes associated with planned vaginal versus planned primary cesarean delivery. Journal of Perinatology. 2010 Apr; 30 (4): 258.

[26] Fidanovski D, Milev V, Sajkovski A, Hristovski A, Sofijanova A, Kojić L, Kimovska M: Mortality risk factors in premature infants with respiratory distress syndrome treated by mechanical ventilation. Srp Arh Celok Lek. 2005 Jan-Feb; 133 (1-2): 29-35.

[27] Miller H. C.: Respiratory Distress Syndrome Of Newborn Infants: Statistical Evaluation of Factors Possibly Affecting Survival of Premature Infants. Pediatrics 1998; 573-579.

[28] Sengupta S, Carrion V, Shelton J, Wynn RJ, Ryan RM, Singhal K, Lakshminrusimha S. Adverse neonatal outcomes associated with early-term birth. JAMA pediatrics. 2013 Nov 1; 167 (11): 1053-9. 